\title{
Model-less/Measurement-based Computation of Voltage Sensitivities in Unbalanced Electrical Distribution Networks
}

\author{
C. Mugnier*, K. Christakou*, J. Jaton ${ }^{\dagger}$, M. De Vivo ${ }^{\dagger}$, M. Carpita ${ }^{\ddagger}$ and M. Paolone* \\ * École Polytechnique Fédérale de Lausanne, Lausanne, Switzerland \\ carl.mugnier@epfl.ch, konstantina.christakou@epfl.ch, mario.paolone@epfl.ch \\ $\dagger$ DEPsys, Yverdon-les-Bains, Switzerland \\ joel.jaton@depsys.ch, michael.devivo@depsys.ch \\ $\ddagger$ Haute École Spécialisée de Suisse Occidentale, Yverdon-les-Bains, Switzerland \\ mauro.carpita@heig-vd.ch
}

\begin{abstract}
Within the context of microgrids optimal voltage control, most schemes proposed in the literature either rely on (i) droop-control methods or (ii) methods involving the computation of explicit nodal power set-points as a solution to a given optimization problem. The first category of approaches is in general suboptimal as it relies on locally sensed measurements. The second category guarantees some level of optimality but requires an accurate and up-to-date model of the network that is, in general, not always available in low voltage grids. To overcome the aforementioned limitations, in this work we propose a methodology suitable for voltage control in generic low voltage 3-phase unbalanced grids. It can be used for the computation of either explicit power set-points or to define the droops of local voltage regulators. Its main characteristic is that it does not rely on the knowledge of the system model and its state. In particular, the goal is to compute linearized dependencies between voltage magnitude and nodal power injections, i.e., voltage sensitivity coefficients. The proposed method assumes availability of a monitoring infrastructure and the computation of the desired sensitivities involves the solution of an over-determined system of linear equations constructed solely using available measurements of nodal power injections and voltage magnitudes. The proposed method is also capable to account for the measurement errors and their time correlation. The performance evaluation of the proposed method is carried out using real measurements coming from a real low voltage feeder located in Switzerland equipped with an appropriate metering infrastructure.
\end{abstract}

Index Terms-Voltage sensitivity coefficients, monitoring infrastructure, power systems optimal operation, voltage control.

\section{INTRODUCTION}

The continuously increasing connection of highly intermittent distributed generation in low voltage grids, essentially composed of renewable energy resources, leads to violations of operational constraints and calls for development of dedicated control mechanisms [1], [2]. In particular, voltage control is one of the typical controls expected to be deployed in distribution systems.

Traditional controls deployed in the case of microgrids mainly rely on droop-control methods (e.g., [3]-[5]). Specifically for the case of voltage control, such methods involve the local sensing of the voltage at the controllable resources connection point and the adjustment of the reactive and/or active power injection of the various resources according to a specific voltage droop characteristic. This category of control provides, in general, suboptimal voltage profiles as it relies on locally available data and does account for the grid topology and parameters. Therefore, such methods lead to suboptimal solutions and, in some cases, to non-feasible operating conditions or even to system collapse [6].

An alternative approach to microgrids voltage control is to directly control the grid by defining explicit set-points for active and reactive nodal power injections (e.g., [1], [7]). These power set-points are typically computed as a solution to an online optimization problem in order to guarantee an optimal grid operation. This category of approaches even though it guarantees some level of optimality compared to the droop-control methods, requires an accurate knowledge of the feeder's topology and parameters. In low voltage grids, this assumption does not always hold in reality. In particular, the distribution network operator (DNO) might have erroneous information on the status of breakers, wrong data for the feeder parameters and the topology is adapted quite frequently [8][10]. Furthermore, there are factors, such as the temperature that can cause variations on the values of the resistances of the network branches along the day and, typically, are not taken into account in the computation of the admittance matrix [11].

In order to overcome the limitations of the aforementioned approaches, in this work we propose a methodology suitable for voltage control in generic low voltage 3-phase unbalanced grids that can be used to compute either explicit power set-points or voltage-droop characteristics of controllable resources but does not rely on the knowledge of the system model. In particular, we are interested in the computation of linearized dependencies between control variables (power injections) and controlled quantities (voltages), i.e., voltage sensitivity coefficients (e.g., [12]-[16]).

The computation of voltage sensitivities requires in general the knowledge of the network topology and parameters. However, recently there has been an effort in the literature to compute sensitivity coefficients using only measurements and, 
thus, avoiding the use of the network admittance matrix. In particular, in [17], [18] a least squares method is proposed for the computation of injection shift factors in transmission networks where large sets of synchronized measurements of PMUs are available. In the same direction, in [19], availability of measurements coming from smart meters is assumed and voltage sensitivity coefficients are computed for a low voltage grid and for different loading scenarios of the network. However, measurements coming from PMUs are, in general, not available in low voltage grids and the noise should also be suitably considered in the computation.

In this paper, we propose a methodology for the computation of voltage sensitivity coefficients in a low voltage 3phase unbalanced grid without relying on the knowledge of the system model and its state. We obtain the desired sensitivities as a solution to an overdetermined system of linear equations. Contrary to the work in [17], [18], we do not require highly synchronized phasor measurements from PMUs, instead we assume availability of a monitoring infrastructure that provides measurements of power injections and voltage magnitudes only. Compared to [19], in this work, we compute sensitivities in the generic case of 3-phase unbalanced networks and we consider the presence of errors in the available measurements, the time correlation of which we take into account in the problem formulation. Furthermore, we perform the computation in an online fashion, thus enabling, in principle, the adoption of the method into real time controllers.

The structure of this paper is the following. In Section II we focus on the problem formulation by describing, in detail, the analytical procedure used for the voltage sensitivities computation relying solely on measurements. In Section III, we describe the case study used for the validation of the proposed method which consists of a real low voltage feeder located in Switzerland, equipped with the necessary monitoring infrastructure. Section IV focuses on the performance evaluation of the proposed method. Finally, Section V provides the main observations and possible directions for future work.

\section{Problem Formulation}

In this section we propose a method for the computation of voltage sensitivities relying solely on measurements, without using any information on the grid model.

As discussed in the literature, the coefficients of interest are the voltage magnitude sensitivities of the $i$-th bus with respect to absorbed/injected power of a bus $j$ defined as:

$$
K_{P i j} \triangleq \frac{\partial E_{i}}{\partial P_{j}} ; K_{Q i j} \triangleq \frac{\partial E_{i}}{\partial Q_{j}}
$$

The computed sensitivities allow for a local linearization of the voltage deviation as a function of the nodal power variations:

$$
\Delta E_{i} \approx \mathbf{K}_{\mathbf{P}_{i}} \boldsymbol{\Delta} \mathbf{P}+\mathbf{K}_{\mathbf{Q}_{i}} \Delta \mathbf{Q} \triangleq\left(\mathbf{K}_{\mathbf{P}, \mathbf{Q}} \boldsymbol{\Delta}(\mathbf{P}, \mathbf{Q})\right)_{i}
$$

where $\mathbf{K}_{\mathbf{P}_{i}}=\left[K_{P_{i 1}}, \ldots, K_{P_{i N}}\right], \mathbf{K}_{\mathbf{Q}_{i}}=\left[K_{Q_{i 1}}, \ldots, K_{Q_{i N}}\right]$, are the vectors of voltage sensitivities of bus $i$.
Such a linearized dependency can be used by the DNO to formulate an optimal control problem whose solution is optimal required nodal power adjustments, which lead to the desired operation set-point for voltage control (e.g., [1], [20]). For instance, the DNO may wish to minimize the voltage deviations from the network rated value $\left(E_{r}\right)$ while respecting the capability curves $(\mathcal{H})$ of a number $N_{D E R}$ of controllable energy resources. In this case, the optimal control problem can be formulated making use of the computed sensitivity coefficients as follows:

$$
\min _{\boldsymbol{\Delta}(\mathbf{P}, \mathbf{Q})} \sum_{i}\left(E_{i}+\left(\mathbf{K}_{\mathbf{P}, \mathbf{Q}} \boldsymbol{\Delta}(\mathbf{P}, \mathbf{Q})\right)_{i}-E_{r}\right)^{2}
$$

subject to:

$$
\left(P_{j}, Q_{j}\right) \in \mathcal{H}_{j}, \quad j=1, \ldots, N_{D E R}
$$

Alternatively, their knowledge can be used for the on-line tuning of droop controllers of flexible resources as a function of the system state. In this case, specific control laws can be designed on the basis of the computed sensitivities, such that the controllable energy resources locally sense the voltage and adjust their power injections in order to guarantee a network voltage profile for safe grid operation.

The sensitivities of interest are typically acquired through an updated Jacobian matrix derived from the load flow problem, via methods based on the use of the so-called adjoint network or using analytical approaches that involve the solution of linear systems of equations (e.g., [12]-[14], [20]-[24]). Despite their differences, all the aforementioned methods require the knowledge of the network admittance matrix. In this work, we use as benchmark the coefficients computed using the method presented in [20].

In this work, in order to estimate the aforementioned voltage sensitivity coefficients using measurements only we rely on the following hypotheses:

H1. The DNO has no knowledge of the network admittance matrix [Y] and system state, i.e., nodal voltage phasors.

$\mathrm{H} 2$. A monitoring infrastructure is available providing the DNO with measurements at frequent time-intervals (in our case sampling frequency is $1 \mathrm{~s}$ ) of the voltage magnitude of each network bus $i,\left(\tilde{E}_{i}(t)\right)$ and of the nodal power injections $\left(\tilde{P}_{i}(t), \tilde{Q}_{i}(t)\right)^{1}$. Note that we do not require the measurements to be highly synchronized as availability of PMUs is still limited in distribution grids and we rely on conventional metering devices ${ }^{2}$. A reasonable assumption is that metering devices are aligned with the network time protocol (NTP) ( [25]).

H3. The desired sensitivities do not vary significantly over a time window of duration $\tau$ during which an adequate number of measurements can be obtained for their computation.

\footnotetext{
${ }^{1}$ In the rest of the paper we denote with a tilde the quantities that correspond to measurements, e.g., $\tilde{E}$.

${ }^{2}$ It is worth noting that, in the absence of a dedicated metering infrastructure, the proposed method can still be deployed, for instance using measurements coming from smart meters that are already present in distribution systems. Alternatively, the method can be used off-line by using pseudomeasurements produced by historic data of nodal power injections.
} 
The key idea behind the proposed method is to use the available measurements in order to compute variations of the voltage magnitudes and corresponding variations of the nodal power injections ${ }^{3}$. In order to be able to compare the obtained measurement-based coefficients with the analytical method in [20], we assume that the nodal power injections are constant power ones, i.e., voltage independent. However, it is worth mentioning that the proposed measurement-based method can be used also in the cases where loads/injections exhibit voltage dependency. Then using the computed variations, a system of linear equations can be obtained starting from (2) that we can solve to obtain the desired coefficients.

In particular, between two consecutive sets of measurements available at time $t$ and $t+\Delta t$ (for a small $\Delta t>0$ ), we define $\Delta \tilde{P}_{i}(t)=\tilde{P}_{i}(t+\Delta t)-\tilde{P}_{i}(t)$ and $\Delta \tilde{Q}_{i}(t)=\tilde{Q}_{i}(t+$ $\Delta t)-\tilde{Q}_{i}(t)$. Similarly for the voltages, the desired variation is computed as $\Delta \tilde{E}_{i}(t)=\tilde{E}_{i}(t+\Delta t)-\tilde{E}_{i}(t)$. If we have a large number of available measurements over a given time window $\tau=\left[t_{1}, t_{m}\right]$ and we make the assumption that the desired sensitivities do not vary significantly during this time period then we can construct the following system of linear equations for each network bus $i$ :

$$
\begin{gathered}
\left(\begin{array}{c}
\Delta \tilde{E}_{i}\left(t_{1}\right) \\
\vdots \\
\Delta \tilde{E}_{i}\left(t_{m}\right)
\end{array}\right) \approx \\
\left(\begin{array}{cccccc}
\Delta \tilde{P}_{1}\left(t_{1}\right) & \cdots & \Delta \tilde{P}_{N}\left(t_{1}\right) & \Delta \tilde{Q}_{1}\left(t_{1}\right) & \cdots & \Delta \tilde{Q}_{N}\left(t_{1}\right) \\
\vdots & \ddots & \vdots & \vdots & \ddots & \vdots \\
\Delta \tilde{P}_{1}\left(t_{m}\right) & \cdots & \Delta \tilde{P}_{N}\left(t_{m}\right) & \Delta \tilde{Q}_{1}\left(t_{m}\right) & \cdots & \Delta \tilde{Q}_{N}\left(t_{m}\right)
\end{array}\right) \cdot\left(\begin{array}{c}
K_{P_{i 1}} \\
\vdots \\
K_{P_{i N}} \\
K_{Q_{i 1}} \\
\vdots \\
K_{Q_{i N}}
\end{array}\right) \\
\\
\end{gathered}
$$

The additional vector $\omega$ in (5) contains the errors from the measurements. These errors are a combination of the measurement errors for both voltages and powers. Among these two, we assume that the effect of the errors linked to the power measurements is negligible compared to the one of the errors in voltage measurements. In order to take into account the voltage measurement noise, we first use a pre-filtering of the acquired measurements. In particular, for each time-step $t$, at least one value of the $\Delta \tilde{E}_{i}(t)$ among all the network buses should be higher than a pre-specified threshold. The value of this threshold is determined based on the uncertainty of the voltage sensors. To fix ideas, this threshold can be $3 \sigma_{E}$, where $\sigma_{E}$ is the variance of a type II uncertainty of a voltage meter. After the filtering, in order to maintain an acceptable number of values that will allow the solution of the problem,

\footnotetext{
${ }^{3}$ Note that the method described next is generic and can be applied to the case of unbalanced networks as it treats each phase of the network separately.
}

the filtered values are replaced by older measurements that satisfy the criterion.

Furthermore, in order to properly model the noise in (5) we take into account the correlation of the errors on the voltage measurements between consecutive time steps. The errors of the voltage measurements are considered gaussian, independent and identically distributed (i.i.d.) with a standard deviation that reflects the accuracy of the metering equipment ${ }^{4}$. However, in (5) we formulate the problem using voltage differences and therefore the noise term $\omega$ exhibits correlation between two consecutive time steps that cannot be neglected.

In particular, the voltage measurement of bus $i$ at time-step $t$ is denoted as:

$$
\tilde{E}_{i}(t)=E_{i}(t)+\epsilon_{i}(t)
$$

where each $\epsilon_{i} \sim \mathcal{N}\left(0, \sigma_{E}\right)$. The errors associated with the voltage measurements are assumed i.i.d between different time steps and different buses.

Using this notation the voltage differences for each bus $i$ are expressed as:

$$
\begin{aligned}
\Delta \tilde{E}_{i}(t+\Delta t) & =\tilde{E}_{i}(t+\Delta t)-\tilde{E}_{i}(t) \\
& =E_{i}(t+\Delta t)-E_{i}(t)+\epsilon_{i}(t+\Delta t)-\epsilon_{i}(t) \\
& =\Delta E(t+\Delta t)+\omega_{i}(t+\Delta t)
\end{aligned}
$$

where $\omega_{i}(t+\Delta t) \triangleq \epsilon_{i}(t+\Delta t)-\epsilon_{i}(t) \sim \mathcal{N}(0, \sqrt{2} \sigma)$ is still gaussian as the difference of 2 gaussian variables but exhibits correlation. The correlation coefficient between two consecutive time-steps is defined as:

$$
\rho\left(\omega_{i}(t), \omega_{i}(t+\Delta t)\right)=\frac{\operatorname{cov}\left(\omega_{i}(t), \omega_{i}(t+\Delta t)\right.}{\sigma_{\omega_{i}(t), \omega_{i}(t+\Delta t)}}
$$

where $\operatorname{cov}\left(\omega_{i}(t), \omega_{i}(t+\Delta t)\right)$

$$
\begin{aligned}
& =\mathbb{E}\left[\left(\omega_{i}(t)-\mathbb{E}\left[\omega_{i}(t)\right]\right)\left(\omega_{i}(t+\Delta t)-\mathbb{E}\left[\omega_{i}(t+\Delta t)\right]\right)\right] \\
& =\mathbb{E}\left[\left(\omega_{i}(t)\right)\left(\omega_{i}(t+\Delta t)\right)\right] \\
& =\mathbb{E}\left[\left(\epsilon_{i}(t)-\epsilon_{i}(t-\Delta t)\right)\left(\epsilon_{i}(t+\Delta t)-\epsilon_{i}(t)\right)\right] \\
& =\mathbb{E}\left[-\epsilon_{i}(t)^{2}\right]=-\sigma^{2}
\end{aligned}
$$

Therefore: $\rho\left(\omega_{i}(t), \omega_{i}(t+\Delta t)\right)=\frac{-\sigma^{2}}{\sqrt{2} \sigma \sqrt{2} \sigma}=-\frac{1}{2}$

Note that, due to the i.i.d and zero-mean assumptions on the errors $\epsilon_{i}$, it holds that $\mathbb{E}\left[\left(\epsilon_{i}(t+k \Delta t) \epsilon_{i}(t+\mu \Delta t)\right)\right]=0, \forall k \neq \mu$. Therefore, the correlation coefficient of the errors $\omega_{i}$ between two non-consecutive time-steps is equal to 0 and the resulting correlation matrix has the following structure ${ }^{5}$ :

$$
\Sigma=\left(\begin{array}{ccccc}
1 & -0.5 & & & \\
-0.5 & \ddots & \ddots & 0 & \\
& \ddots & \ddots & \ddots & \\
& 0 & \ddots & \ddots & -0.5 \\
& & & -0.5 & 1
\end{array}\right)
$$

\footnotetext{
${ }^{4}$ Note that by metering equipment in this section we refer to industrialgrade metering infrastructure.

${ }^{5}$ Note that $\Sigma$ is correct for the ideal case where the errors in the power measurements are less dominant than those of the voltages. If both errors need to be accounted for, $\Sigma$ is not known a priori and its assessment might require a more sophisticated analysis.
} 


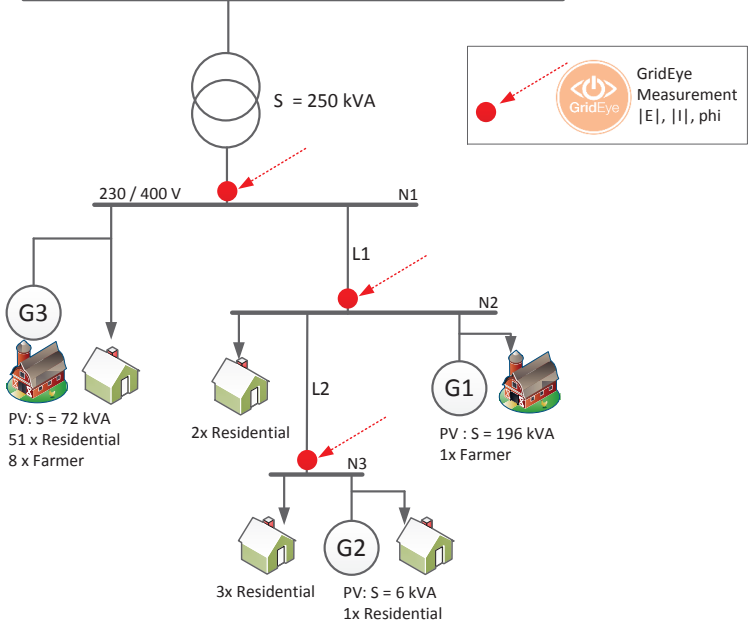

Figure 1. Real low voltage distribution feeder used for the performance evaluation of the proposed method.

Table I

LINES PARAMETERS

\begin{tabular}{|l|l|c|c|c|}
\hline & Cable type & Length & $(\mathbf{R}, \mathbf{X}) \mathbf{O h m} / \mathbf{k m}$ & $\mathbf{C}$ uF/km \\
\hline L1 & $1 \mathrm{kV} 4 \times 240 \mathrm{~mm} 2 \mathrm{AL}$ & $219 \mathrm{~m}$ & $(0.096,0.072)$ & 0.77 \\
\hline L2 & $1 \mathrm{kV} 4 \times 150 \mathrm{~mm} 2 \mathrm{AL}$ & $145 \mathrm{~m}$ & $(0.2633,0.078)$ & 0.73 \\
\hline
\end{tabular}

Provided that $t_{m}>2 N$, we have formulated the problem as an over-determined system of linear equations that can be solved using a generalized least squares method to account also for the correlated errors. In such a case, the sensitivity coefficients are obtained analytically through the resolution of the following equations [26]:

$\mathbf{K}_{\mathbf{P Q}}=\left(\boldsymbol{\Delta}(\tilde{\mathbf{P}}, \tilde{\mathbf{Q}})_{\tau}^{\mathbf{T}} \boldsymbol{\Sigma}^{-1} \boldsymbol{\Delta}(\tilde{\mathbf{P}}, \tilde{\mathbf{Q}})_{\tau}\right)^{-1} \boldsymbol{\Delta}(\tilde{\mathbf{P}}, \tilde{\mathbf{Q}})_{\tau}^{\mathbf{T}} \boldsymbol{\Sigma}^{-1} \Delta \tilde{\mathbf{E}}_{\mathbf{i}, \tau}$

It is important to note that when the pre-filtering described earlier is considered, then certain columns (rows) of the matrix $\Delta(\tilde{\mathbf{P}}, \tilde{\mathbf{Q}})_{\tau}\left(\Delta \tilde{\mathbf{E}}_{\mathbf{i}, \tau}\right)$ are removed. In this case the correlation matrix needs to be adjusted properly setting to zero the entries that do not correspond to measurements of consecutive time steps.

\section{CASE Study}

In this section we present the case study used for the evaluation of the proposed method. We provide all the details of the grid topology, as well as the relevant metering infrastructure.

\section{A. Network Configuration}

The network used in this case study is a real low voltage three-phase radial distribution feeder $(230 / 400 \mathrm{~V}, 50 \mathrm{~Hz})$ located in a rural area in Switzerland, shown in Fig. 1. This particular feeder is composed of 57 residential blocks, 9 agricultural buildings and supplies in total 88 customers. The characteristics of the feeder and the substation transformer are summarized in Table I and Table II.
Table II

TRANSFORMER PARAMETERS

\begin{tabular}{|c|c|c|c|c|c|c|}
\hline & Power & $\mathbf{U}_{\text {in }}$ & $\mathbf{U}_{\text {out }}$ & Coupling & $\mathbf{U}_{\mathbf{c c}}$ & $\mathbf{X} / \mathbf{R}$ \\
\hline $\mathrm{T} 1$ & $250 \mathrm{kVA}$ & $20 \mathrm{kV}$ & $230 / 400 \mathrm{~V}$ & DYn11 & $4.1 \%$ & 2.628 \\
\hline
\end{tabular}

Table III

PV GENERATORS CHARACTERISTICS

\begin{tabular}{|c|c|c|}
\hline PV Generators & Number of inverters & Rated Power (kVA) \\
\hline G1 & 12 3-phase & 196 \\
\hline G2 & 2 1-phase & 6 \\
\hline G3 & 3 3-phase & 72 \\
\hline
\end{tabular}

This system has been selected as it contains non-negligible injections from photovoltaic systems. The existing decentralized PV plants (marked G1, G2 and G3 in Fig. 1) provide a maximum power of $274 \mathrm{kVA}$ and their characteristics are reported in Table III. With the existing PV capacity, there are time-periods when the production of power is larger than the consumption of the entire feeder. The associated power flows in these cases cause non-negligible voltage fluctuations above the allowed limits. In particular, voltage variations of $9.1 \%$ larger that the network rated value are constantly observed with consequent impacts on the quality of service.

The second reason why this grid was selected is because its network topology and component data are available and, therefore, it is feasible to validate the proposed method by comparing the assessment of the measurement-based sensitivities with the benchmark ones computed using the network admittance matrix.

\section{B. Measurements}

This particular feeder is equipped with metering devices called GridEye ${ }^{6}$. These devices measure, for each of the three phases, the voltage and the input current at each network node with a high sampling frequency $(50 \mathrm{kHz})$. Once the measurements are acquired, a post-processing of the measured quantities is performed using an Interpolated-Modulated DFT ( [27]-[29]) on an embedded ARM processor that allows the computation of the nodal (phase-to-ground) real and reactive power, as well as the voltage magnitude.

The specifications of the metering equipment, as well as the specific accuracies related to the voltage, current and phase measurements are reported below in Table IV.

\section{PERformanCE ASSESSMENT}

The numerical validation of the proposed method is carried out using the real measurements coming from the real threephase LV feeder in Switzerland described in the previous section.

In order to be able to compare the performances of the proposed method with the formal analytic method presented in [20] we need to have access to the true grid state and the

\footnotetext{
${ }^{6}$ GridEye is a LV grid metering and control tool, which has been developed by the DEPsys Company and is used in this paper for the metering function only.
} 
true topological information that correspond to the obtained measurements. To this end, we adopt the following procedure. We consider the nodal power profiles given by a set of real measurements ${ }^{7}$. Fig. 2-4 show the active and reactive power measurements of phases $a, b$ and $c$ of buses 1,2 and 3 during a time window of $9.22 \mathrm{~h}$. Moreover, we use the nominal values of the network admittance matrix that, for the sake of validation, is available and is computed using the network data reported in Tables I,II. Using this data, we perform a threephase load flow calculation to obtain the voltage phasors that correspond to the nominal admittance matrix and the measured power profiles. This provides us with the ground truth and allows the computation of the exact sensitivity coefficients. Next, we use the load-flow voltage magnitude profiles and we create a set of pseudo-measurements by adding white Gaussian noise with a standard deviation of $2.17 E-4$ that represents the accuracy of the metering equipment ${ }^{8}$. This set of pseudomeasurements, along with the true measurements of the nodal power injections, are used as input for the measurement-based computation of the sensitivity coefficients.

For the sake of brevity, only a few coefficients will be shown for every part of the validation process. Note that cross-phase coefficients are not shown in what follows. These coefficients are zero in this case-study as the grid topology is symmetric despite the imbalances in the network loads. In Fig. 5 and 6 we emphasize the importance of the size of the time window used for the estimation. The red solid line represents the actual voltage coefficients of phase $a$ of bus 3 with respect to the active power injection of phase $a$ of the

Table IV

Metering Devices Characteristics

\begin{tabular}{|l|c|c|c|}
\hline $\begin{array}{c}\text { Voltage } \\
\text { measurements }\end{array}$ & $\begin{array}{c}3 \text { isolated inputs } \\
\text { (3L-N), } \\
\text { High speed } 16 \text { bits } \\
\text { ADC sampling }\end{array}$ & $\begin{array}{c}\text { Reading Resolution: } \\
0.05 V_{R M S} \\
\text { Accuracy: } \\
\pm 0.05 V_{R M S} \\
\text { of RMS value } \\
\text { (maximum } \\
\text { absolute error) }\end{array}$ & $1-300 V_{R M S}$ \\
\hline $\begin{array}{c}\text { Current } \\
\text { measurements }\end{array}$ & $\begin{array}{c}\text { 4 inputs with } \\
\text { Rogowski coil } \\
\text { ADC sampling } 16 \text { bits } \\
1 A_{R M S} \\
\text { Accuracy: } \\
\pm(1 \% \text { of } \\
\text { measured value }+ \\
0.025 \% \text { of range) }\end{array}$ & $1-1500 A_{R M S}$ \\
\hline Phase shift & $\begin{array}{c}\text { Resolution: 1 degree. } \\
\text { Accuracy: 1 degree }\end{array}$ & $0-360^{\circ}$ \\
\hline $\begin{array}{l}\text { Sync system } \\
\text { actual version }\end{array}$ & NTP based on GSM communication \\
\hline $\begin{array}{l}\text { Sync system } \\
\text { new version }\end{array}$ & \multicolumn{2}{|l|}{ NTP, PTP, GPS or DCF 77 } \\
\hline
\end{tabular}

\footnotetext{
${ }^{7}$ In this study we use measurement-traces acquired during February and July to capture the different loading conditions of the grid during different seasons.

${ }^{8}$ For the computation of the standard deviation corresponding to the voltage measurements we have used the maximum absolute error of $50 \mathrm{mV}$ reported in Table IV divided by the nominal network rated voltage value as all the computations are performed in p.u. quantities.
}

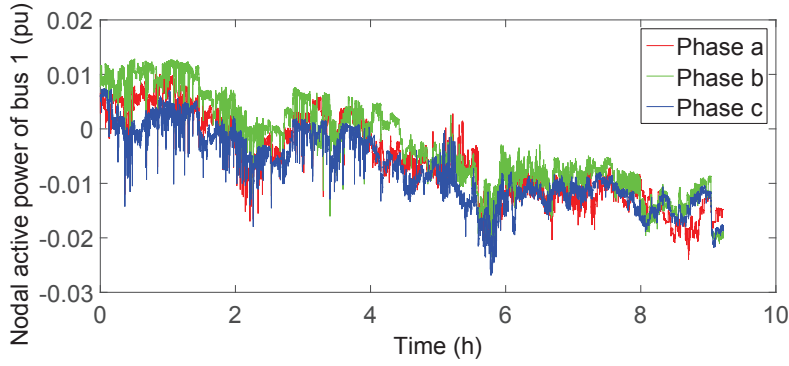

(a) Nodal active power measurements (p.u.) of bus 1 .

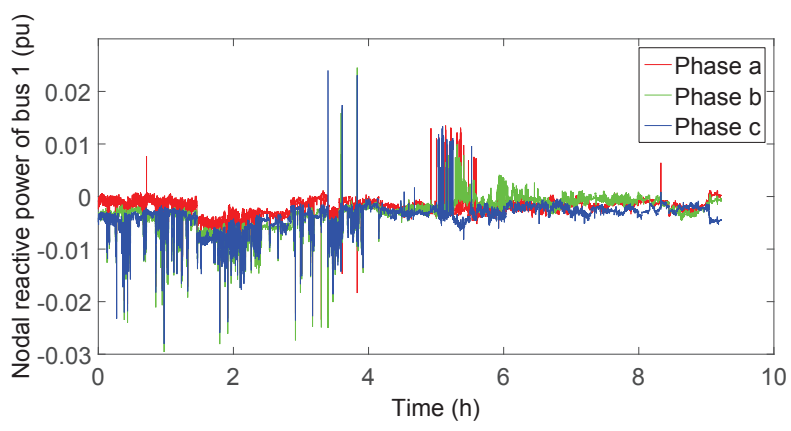

(b) Nodal reactive power measurements (p.u.) of bus 1 .

Figure 2. Nodal power measurements (p.u.) of bus 1.

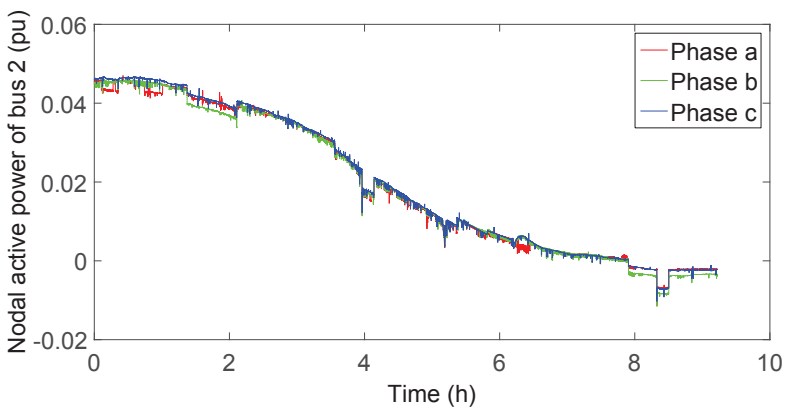

(a) Nodal active power measurements (p.u.) of bus 2 .

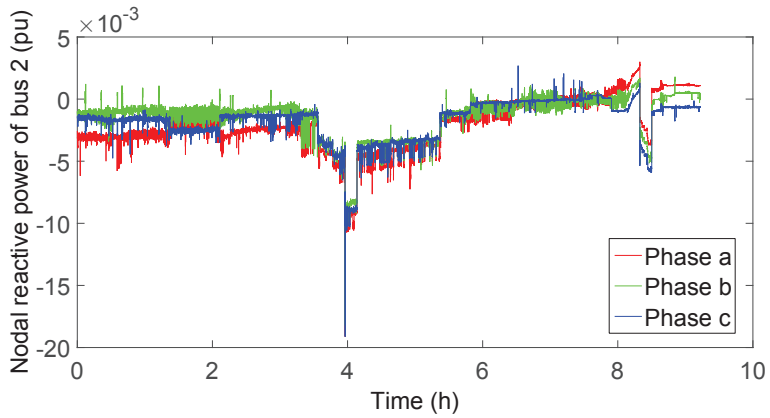

(b) Nodal reactive power measurements (p.u.) of bus 2 .

Figure 3. Nodal power measurements (p.u.) of bus 2.

same bus. The blue and green curves depict the corresponding measurement-based coefficients using a time window of $200 \mathrm{~s}$ and 1000 s respectively, whilst the red curve corresponds to the true coefficients computed using [20]. It is worth observing that even though both the blue and green curves are quite close to the actual coefficients in the first $1 \mathrm{~h}$, the blue curve exhibits large variations in the last $1.5 \mathrm{~h}$. The reason for this is that the least squares problem that needs to be solved is badly 


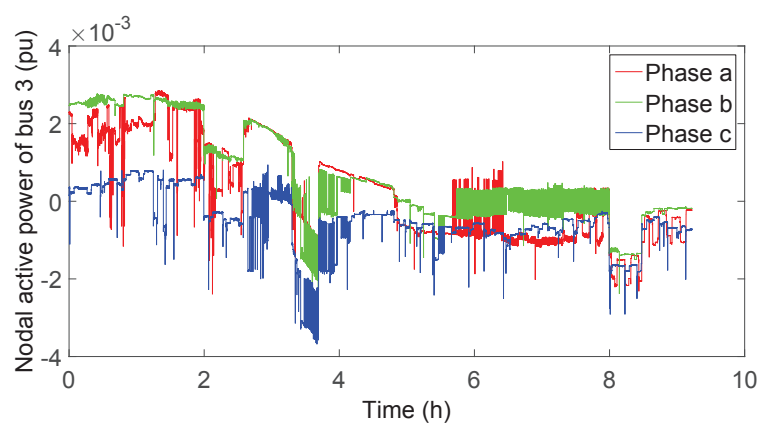

(a) Nodal active power measurements (p.u.) of bus 3 .

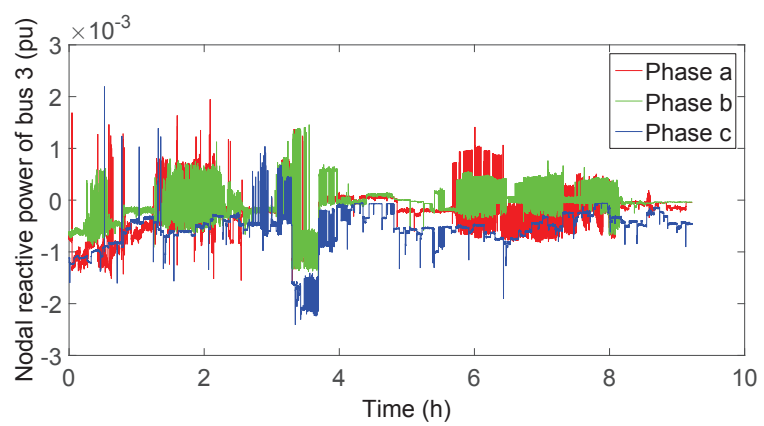

(b) Nodal reactive power measurements (p.u.) of bus 3 .

Figure 4. Nodal power measurements (p.u.) of bus 3 .

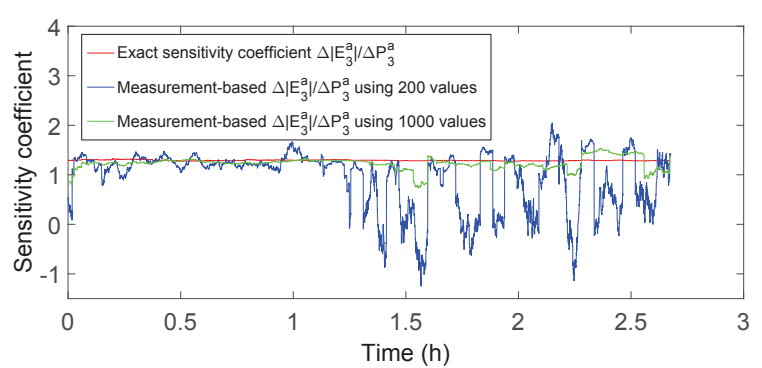

Figure 5. Exact and measurement-based voltage sensitivity coefficients of bus 3 phase $a$ with respect to the active power of bus 3 phase $a$ using different window sizes.

conditioned. This is shown in Fig. 6 where it can be observed that with a time window of $200 \mathrm{~s}$, the condition number of the matrix that needs to be inverted increases significantly and consequently the quality of the estimated coefficients becomes worse compared to the case of a $1000 \mathrm{~s}$ time-window. Therefore, in what follows we choose to estimate the desired coefficients using a measurement time-window of $2000 \mathrm{~s}^{9}$.

Fig. 7-9 show the exact voltage sensitivities in red line, the measurement-based sensitivities without the noise prefiltering described in Section II in green and the measurementbased sensitivities using the noise pre-filtering in blue. In particular, Fig. 7 shows the sensitivity of phase $c$ of bus 2 w.r.t. active power of phase $c$ of bus 2, Fig. 8 shows the sensitivity of phase $a$ of bus 3 w.r.t. active power of phase $a$ of bus 3 and Fig. 9 shows the sensitivity of phase $b$ of bus 3 w.r.t. reactive power of phase $b$ of bus 3 . In all

\footnotetext{
${ }^{9}$ The final size of the time-window was decided by performing a series of simulation experiments with different time-windows varying from $200 \mathrm{~s}$ to $5000 \mathrm{~s}$ and keeping the smallest value which did not result in large condition numbers of the least squares matrix.
}

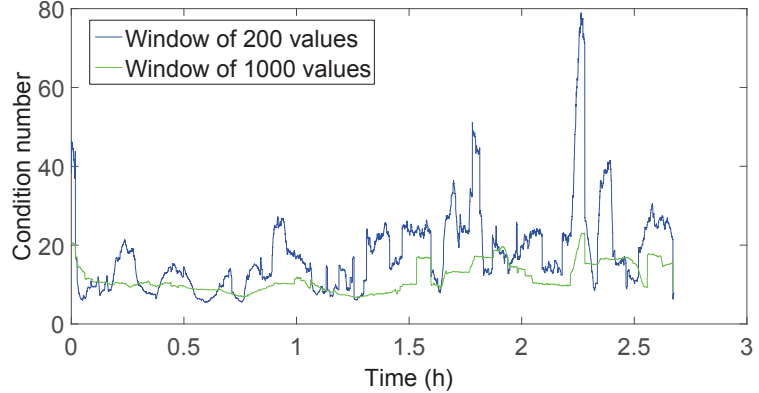

Figure 6. Condition number of the matrix used in the least-squares problem for different window sizes.

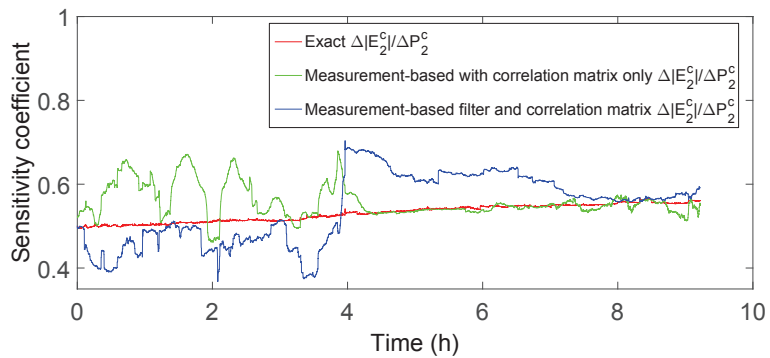

Figure 7. Exact and measurement-based voltage sensitivity coefficients of bus 2 phase $c$ with respect to the active power of bus 2 phase $c$ using solely correlation or noise pre-filtering and correlation.

cases, the measurement-based estimates of the coefficients are close to the exact values computed using the analytical method in [20]. However, it is worth noting that in some cases not using the pre-filtering of the noise (green curves) results in significant peaks in the estimated coefficients (Fig. 8) that can also lead to values of the sensitivities that are very far away from the actual coefficients (for instance negative values in Fig. 9). This behavior is observed when the network state does not vary significant from one time step to the next, and

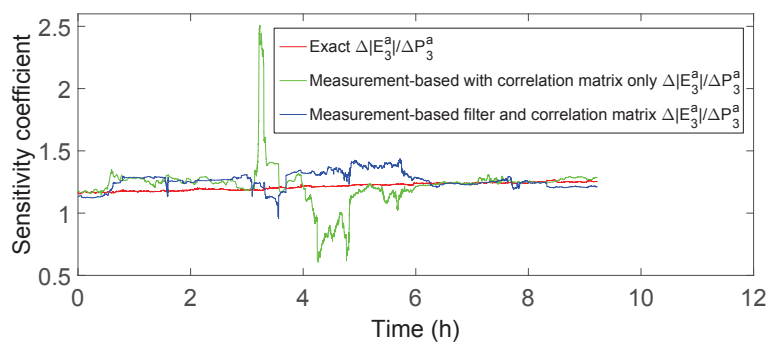

Figure 8. Exact and measurement-based voltage sensitivity coefficients of bus 3 phase $a$ with respect to the active power of bus 3 phase $a$ using solely correlation or noise pre-filtering and correlation.

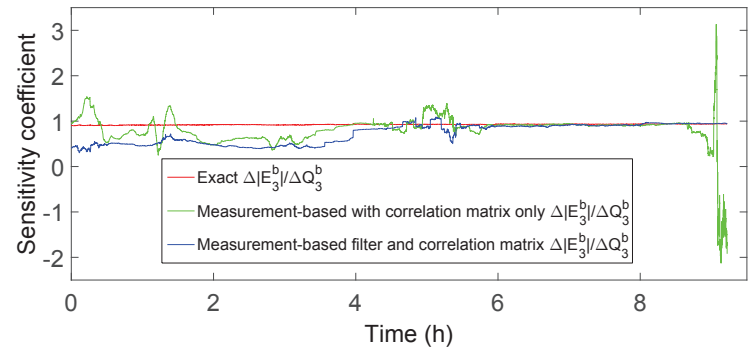

Figure 9. Exact and measurement-based voltage sensitivity coefficients of bus 3 phase $b$ with respect to the reactive power of bus 3 phase $b$ using solely correlation or noise pre-filtering and correlation. 
therefore the matrices corresponding to the voltage and power differences are essentially composed of noise and lead to an ill-conditioned system (time $3.2 \mathrm{~h}$ in Fig. 8 or time $9.1 \mathrm{~h}$ in Fig. 9). In this case, using the pre-filtering technique described earlier leads to much better estimates of the sensitivities as evidenced by the blue curves in Fig. 8 and 9 which are much closer to the exact coefficients and do not exhibit large variations across the time-steps.

\section{CONCLUSION}

In this paper we have proposed a methodology suitable for generic low voltage 3-phase unbalanced grids that can be used to supply either a centralized optimal control or for the definition of time-variant voltage-droop characteristics. The method does not rely on the knowledge of the network admittance matrix. In particular, we have computed voltage sensitivities as a solution to an over-determined system of linear equations constructed solely using measurements of nodal power injections and voltage magnitudes. We have considered the presence of errors in the available measurements, the time correlation of which we have taken into account explicitly in the problem formulation. The proposed method has been validated and its performance has been evaluated using real measurements coming from a low voltage feeder located in Switzerland equipped with an industrial-grade metering infrastructure. The proposed method represents the foundation of a more generic approach in which power uncertainties and their time correlation can be considered. In this case, a suitable computation of the correlation matrix $\Sigma$ is required. Future work will be focused on this specific aspect.

\section{ACKNOWLEDGEMENTS}

The authors gratefully acknowledge the CTI - Commission for Technology and Innovation (CH), the SFOE - Federal Office of Energy $(\mathrm{CH})$, Romande Energie $(\mathrm{CH})$ and the SCCERFURIES - Swiss Competence Center for Energy Research Future Swiss Electrical Infrastructure, for their financial and technical support to the research activity presented in this paper.

\section{REFERENCES}

[1] A. Borghetti, M. Bosetti, S. Grillo, S. Massucco, C. Nucci, M. Paolone, and F. Silvestro, "Short-term scheduling and control of active distribution systems with high penetration of renewable resources," Systems Journal, IEEE, vol. 4, no. 3, pp. 313-322, 2010.

[2] Q. Zhou and J. Bialek, "Generation curtailment to manage voltage constraints in distribution networks," Generation, Transmission \& Distribution, IET, vol. 1, no. 3, pp. 492-498, 2007.

[3] K. De Brabandere, B. Bolsens, J. Van den Keybus, A. Woyte, J. Driesen, and R. Belmans, "A voltage and frequency droop control method for parallel inverters," IEEE Trans. on Power Electronics, vol. 22, no. 4, pp. 1107-1115, 2007.

[4] P. Piagi and R. Lasseter, "Autonomous control of microgrids," in Power Engineering Society General Meeting, 2006. IEEE, 2006, pp. 8 pp.-.

[5] N. Hatziargyriou, Microgrids: Architectures and Control, J. Wiley and S. Ltd, Eds., 2014.

[6] L. Reyes-Chamorro, A. Bernstein, J.-Y. Le Boudec, and M. Paolone, "A composable method for real-time control of active distribution networks with explicit power setpoints. Part II: Implementation and validation," Electric Power Systems Research, vol. 125, pp. 265-280, 2015.
[7] A. Bernstein, L. Reyes-Chamorro, J.-Y. Le Boudec, and M. Paolone, "A composable method for real-time control of active distribution networks with explicit power setpoints. Part I: Framework," Electric Power Systems Research, vol. 125, pp. 254-264, 2015.

[8] A. Al-Othman and M. Irving, "Analysis of confidence bounds in power system state estimation with uncertainty in both measurements and parameters," Electric power systems research, vol. 76, no. 12, pp. 1011$1018,2006$.

[9] D.-H. Choi and L. Xie, "Impact analysis of locational marginal price subject to power system topology errors," in 2013 IEEE International Conference on Smart Grid Communications (SmartGridComm), Oct 2013, pp. 55-60.

[10] K. Clements and P. Davis, "Detection and identification of topology errors in electric power systems," IEEE Trans. on Power Systems, vol. 3, no. 4, pp. 1748-1753, Nov 1988.

[11] S. Frank, J. Sexauer, and S. Mohagheghi, "Temperature-dependent power flow," IEEE Trans. on Power Systems, vol. 28, no. 4, pp. 40074018, Nov 2013.

[12] J. Peschon, D. Piercy, W. Tinney, and O. Tveit, "Sensitivity in power systems," IEEE Trans. on Power Apparatus and Systems, no. 8, pp. 1687-1696, 1968.

[13] D. Shirmohammadi, H. Hong, A. Semlyen, and G. Luo, "A compensation-based power flow method for weakly meshed distribution and transmission networks," IEEE Trans. on Power Systems, vol. 3, no. 2, pp. 753-762, 1988.

[14] A. Wood and B. Wollenberg, Power generation, operation, and control. Wiley New York, 1996, vol. 2.

[15] R. Marconato, "Electric power systems, vol. 2," CEI, Italian Electrotechnical Committee, Milano, Italy, 2002.

[16] M. Begovic and A. Phadke, "Control of voltage stability using sensitivity analysis," IEEE Trans. on Power Systems, vol. 7, no. 1, pp. 114-123, 1992.

[17] Y. Chen, A. Dominguez-Garcia, and P. Sauer, "A sparse representation approach to online estimation of power system distribution factors," IEEE Trans. on Power Systems, vol. PP, no. 99, pp. 1-12, 2014.

[18] - "Measurement-based estimation of linear sensitivity distribution factors and applications," IEEE Trans. on Power Systems, vol. 29, no. 3, pp. 1372-1382, May 2014.

[19] S. Weckx, R. D'Hulst, and J. Driesen, "Voltage sensitivity analysis of a laboratory distribution grid with incomplete data," IEEE Trans. on Smart Grid, vol. 6, no. 3, pp. 1271-1280, May 2015.

[20] K. Christakou, J.-Y. LeBoudec, M. Paolone, and D.-C. Tomozei, "Efficient computation of sensitivity coefficients of node voltages and line currents in unbalanced radial electrical distribution networks," IEEE Trans. Smart Grid, vol. 4, no. 2, pp. 741-750, 2013.

[21] Q. Zhou and J. Bialek, "Simplified calculation of voltage and loss sensitivity factors in distribution networks," in Proc. of the 16th Power Systems Computation Conference (PSCC2008), Glasgow, Scotland, 2008.

[22] J. Bandler and M. El-Kady, "A unified approach to power system sensitivity analysis and planning, part I: Family of adjoint systems," in Proc. IEEE Int. Symp. Circuits Syst, 1980, pp. 681-687.

[23] — , "A new method for computerized solution of power flow equations," IEEE Trans. on Power Apparatus and Systems, no. 1, pp. 1-10, 1982.

[24] — "A unified approach to power system sensitivity analysis and planning, part II: Special class of adjoint systems," in Proc. IEEE Int. Symp. Circuits Syst, 1980, p. 688.

[25] D. L. Mills, "Internet time synchronization: the network time protocol," IEEE Trans. Communications, vol. 39, no. 10, pp. 1482-1493, 1991.

[26] T. Kariya and H. Kurata, Generalized least squares. John Wiley \& Sons, 2004.

[27] P. Romano and M. Paolone, "Enhanced interpolated-dft for synchrophasor estimation in fpgas: Theory, implementation, and validation of a pmu prototype," IEEE Trans. on Instrumentation and Measurement, vol. 63, no. 12 , pp. 2824-2836, 2014.

[28] P. Romano, M. Paolone, J. Arnold, and R. Piacentini, "An interpolated$\mathrm{dft}$ synchrophasor estimation algorithm and its implementation in an fpga-based pmu prototype," in Power and Energy Society General Meeting (PES), 2013 IEEE. IEEE, 2013, pp. 1-6.

[29] P. Romano and M. Paolone, "An enhanced interpolated-modulated sliding dft for high reporting rate pmus," in 2014 IEEE International Workshop on Applied Measurements for Power Systems Proceedings (AMPS). IEEE, 2014, pp. 1-6. 